\title{
Underlying mechanism of the photodynamic activity of hematoporphyrin-induced apoptosis in U87 glioma cells
}

\author{
SHI-XIANG YUAN ${ }^{1,2}$, JUN-LIANG LI $^{3}$, XIN-KE XU $^{3}$, WEI CHEN $^{3}, \mathrm{CHENG} \mathrm{CHEN}^{3}$, \\ KUN-QI KUANG ${ }^{4}$, FANG-YU WANG ${ }^{3}, \mathrm{KAI} \mathrm{WANG}^{2}$ and FANG-CHENG $\mathrm{LI}^{3}$ \\ ${ }^{1}$ The First Clinical Medical College, Jinan University, Guangzhou, Guangdong 510632; \\ ${ }^{2}$ Department of Neurosurgery, The Twelve People's Hospital of Guangzhou City, Guangzhou, Guangdong 510620; \\ ${ }^{3}$ Department of Neurosurgery, Guangzhou Women and Children's Medical Center, Guangzhou Medical University, \\ Guangzhou, Guangdong 510623; ${ }^{4}$ Department of Neurosurgery, Sun Yat-sen Memorial Hospital, \\ Sun Yat-sen University, Guangzhou, Guangdong 510120, P.R. China
}

Received June 13, 2017; Accepted January 9, 2018

DOI: $10.3892 / \mathrm{ijmm} .2018 .3400$

\begin{abstract}
Photodynamic therapy (PDT) is a relatively novel type of tumor therapy method with low toxicity and limited side-effects. The aim of the present study was to investigate the underlying mechanism and potential microRNAs (miRNAs) involved in the treatment of glioma by PDT with hematoporphyrin, a clinical photosensitizer. The photodynamic activity of hematoporphyrin on the cell viability and apoptosis of gliomas was investigated by MTT, and flow cytometry and fluorescence microscopy, respectively. Alterations in singlet oxygen and mitochondrial membrane potential were detected. The differentially expressed miRNAs and proteins were evaluated by miRNA gene chip and apoptosis-associated protein chip, respectively. The results demonstrated that cell viability significantly decreased with hematoporphyrin concentration. PDT with hematoporphyrin significantly increased cell apoptosis at a later stage, induced the content of reactive oxygen species (ROS) and decreased the mitochondrial membrane potential, indicating that PDT with hematoporphyrin inhibited cell growth via induction of radical oxygen, decreased the mitochondrial membrane potential and induced apoptosis. The upregulated miRNAs, including hsa-miR-7641, hsa-miR-9500, hsa-miR-4459, hsa-miR-21-5p, hsa-miR-663a and hsa-miR-205-5p may be important in PDT-induced cell apoptosis in glioma. Transporter 1, ATP binding cassette subfamily B member- and nuclear factor- $\kappa \mathrm{B}$-mediated apoptosis signaling pathways were the most significant
\end{abstract}

Correspondence to: Dr Fang-Cheng Li, Department of Neurosurgery, Guangzhou Women and Children's Medical Center, Guangzhou Medical University, 9 Jinsui Road, Guangzhou, Guangdong 510623, P.R. China

E-mail: sjwklfc@126.com

Key words: photodynamic therapy, hematoporphyrin, miR-21-5p, glioma, apoptosis pathways. Thus, the current study presents PDT as a potential therapeutic approach for the treatment of malignant glioma, and identified miRNAs for the molecular design and development of a third-generation photosensitizer (PS).

\section{Introduction}

Glioma is the most common type of malignant tumor of the central nervous system, accounting for $\sim 46 \%$ of brain tumors $(1,2)$. Glioma is a neuroectodermal tumor with the highest mortality rate of primary intracranial tumors (3). Currently, craniotomy is the most efficient type of therapy to treat gliomas (4). However, due to the invasive growth of gliomas and its unclear demarcation with normal brain tissues, it is difficult to completely remove tumor tissues by surgery, resulting in a high recurrence rate $(5,6)$, and subsequent treatment with radiotherapy and chemotherapy (which damage the immune system) are generally not satisfactory (7). Methods to decrease the high recurrence rates and serious side-effects of glioma treatment are being investigated to develop specific drugs or therapeutic strategies with high efficiency and sensitivity, and low toxicity for the treatment of gliomas (8).

Photodynamic therapy (PDT) is a novel therapeutic method for tumor treatment (9). PDT was first discovered by Dougherty et al (10) in 1900, and was first used in medicine to ablate and destroy unwanted tissue in 1975 (10). PDT utilizes the activation of a photosensitizer (PS) by light irradiation with a specific wavelength to generate oxygen radicals and further inhibit growth of tumor cells $(11,12)$. The advantages of PDT are its minimally invasive characteristic, repeatability, low toxicity accumulation when compared with the traditional treatments, in addition to its low recurrence rate and more definitive outcome (9). Low toxicity to normal tissue is a significant advantage of PDT, compared with traditional treatment. In recent years, PDT has shown a particularly good therapeutic effect on bladder cancer, early lung cancer, Barrett's esophagus, head and neck cancer and skin cancer, amongst others (13-15). For example, when using 2-(1-hexyloxyethyl)-2-devinyl pyropheophorbide-a as a PS in 
the clinical setting, PDT demonstrated a particularly effective outcome in 16 cases of patients with lung cancer in a treatment cycle, with a very slight photosensitive side-effect; after 3 days of PDT treatment, patients with oral cancer recovered (16). Furthermore, PDT treatment for esophageal cancer and Barrett's esophagus resulted in a good outcome (17). However, fundamental difficulties encountered in the treatment of PDT, such as targeting, light source and light dose have not been addressed. Advances in chemistry, materials, optics, clinical treatment and other disciplines contribute to overcoming these difficulties, by elucidating the underlying mechanism of PDT.

Typically, tumor treatment methods involve enhancing the antitumor immune response of the body via immunology. In recent years, PDT was identified as able to inhibit the growth of tumor cells and regulate the conditional body immune system; thus, has gradually been adopted as an auxiliary treatment to reduce the recurrence rate of tumors (18). In previous studies, PDT was identified as a promising treatment strategy for glioma (19-22). Using 5-aminolevulinic acid (5-ALA) as a PS, the expression level of proteasomes was increased in cultured U251 cells and the growth of glioma cells was inhibited under light irradiation (19). Using hematoporphyrin as a PS, PDT induced the transcriptional level of transporter 1, ATP binding cassette subfamily B member (TAPI) and the expression level of HLA-1 in U251 cells under light irradiation, inhibiting the growth of glioma cells in a dose- and time-dependent manner (20). In addition, PDT enhanced the immunogenicity of glioma cells, indicating that a PDT-generated glioma vaccine from dendritic cells presents as a promising novel method for tumor treatment (21). However, the molecular mechanisms underlying PDT-inhibited growth of glioma cells remain unclear. In the current study, based on previous studies, the role of PDT in the induction of cell apoptosis of glioma cells was investigated using hematoporphyrin as the PS, as well as microRNAs (miRNAs) and their corresponding signaling pathways, such as target molecule networks. The current results provide a potential therapeutic strategy involving PDT for the treatment of gliomas.

\section{Materials and methods}

Cell culture. Human glioma cell lines U87 and U251 cells were obtained from the American Type Culture Collection (ATCC; Manassas, VA, USA). Cells were cultured in 5\% $\mathrm{CO}_{2}$ and $95 \%$ humidified atmosphere in DM 1640 medium (Invitrogen, Thermo Fisher Scientific, Inc., Waltham, MA, USA) containing 10\% fetal bovine serum (Invitrogen; Thermo Fisher Scientific Inc.) with $100 \mathrm{U} / \mathrm{ml}$ penicillin and $100 \mu \mathrm{g} / \mathrm{ml}$ streptomycin. Cells were passaged subsequent to reaching $80 \%$ confluence.

MTT assay. Cell viability was determined using MTT. Briefly, $5 \times 10^{3}$ cells per well were seeded in 96-well plates. Following incubation for $12 \mathrm{~h}$ at $37^{\circ} \mathrm{C}$, the cells were treated with phosphate-buffered saline (PBS; control) or various concentrations of hematoporphyrin (0-120 nM; MedChemExpress, Princeton, NJ, USA) for $60 \mathrm{~min}$. Following light irradiation using an M8 Spectrum Power Energy meter (San Diego, Taizhong, Taiwan, China) at $625 \mathrm{~nm}, 5 \mathrm{~mW} / \mathrm{cm}^{2}$ for $60 \mathrm{~min}$ (23) and culturing for $22 \mathrm{~h}$ at $37^{\circ} \mathrm{C}, 100 \mu 1 \mathrm{MTT}$ solution $(5.0 \mathrm{mg} / \mathrm{ml})$ was then added to each well and incubated for $4 \mathrm{~h}$ at $37^{\circ} \mathrm{C}$, followed by removal of culture medium and the addition of dimethyl sulfoxide (100 $\mu \mathrm{l} /$ well) to dissolve the formed formazan crystals. The absorbance value was determined using a microplate reader (Varioskan Flash, Thermo, USA) at a wavelength of $520 \mathrm{~nm}$. The relative cell viability was calculated by normalization to the control. The experiments were independently performed three times.

Cell apoptosis assays. Cell apoptosis was detected using Hoechst 33342 and flow cytometry. Briefly, 2x10 $10^{5}$ cells/well were seeded in 6-well plates. Subsequent to incubation for $12 \mathrm{~h}$ at $37^{\circ} \mathrm{C}$, the cells were treated with hematoporphyrin $(85 \mathrm{nM}$, the $\left.\mathrm{IC}_{50}\right)$ under light irradiation $\left(625 \mathrm{~nm}, 5 \mathrm{~mW} / \mathrm{cm}^{2}\right)$ for $60 \mathrm{~min}$. The untreated cells served as the control. Cells were subsequently cultured for $23 \mathrm{~h}$ at $37^{\circ} \mathrm{C}$, then collected with trypsin-ethylenediaminetetraacetic acid (EDTA) and resuspended in PBS. Cell apoptosis was measured by flow cytometry (BD FACSCalibur; BD Biosciences, Franklin Lakes, NJ, USA) using the Annexin V-fluorescein isothiocyanate (Beyotime Institute of Biotechnology, Haimen, China) according to the manufacturer's instructions. The flow cytometry data was analyzed using FlowJo version 10.0 software (FlowJo LLC, Ashland, OR, USA). For the Hoechst 33342 assay, $10 \mu 1$ Hoechst $33342(5 \mu \mathrm{g} / \mathrm{ml})$ was added to each well and incubated for $30 \mathrm{~min}$ at $37^{\circ} \mathrm{C}$ in the dark, followed by removal of the culture medium and two washes with PBS. The apoptotic nuclei were detected by inverted fluorescence microscopy (SR GSD; Leica Microsystems, Inc., Buffalo Grove, IL, USA).

TUNEL assay. Cells (2x10 /well) were seeded in 12-well plates. After adherence, cells were treated with hematoporphyrin $(85 \mathrm{nM})$ under light irradiation $\left(625 \mathrm{~nm}, 5 \mathrm{~mW} / \mathrm{cm}^{2}\right)$ for $60 \mathrm{~min}$. The untreated cells served as the control. Subsequently, cells were cultured for $23 \mathrm{~h}$ at $37^{\circ} \mathrm{C}$, and fixed with $4 \%$ paraformaldehyde for $30 \mathrm{~min}$, washed with $\mathrm{PBS}$, incubated with $0.3 \%$ Triton X-100 (Solarbio, Shanghai, China) in PBS for $5 \mathrm{~min}$, and incubated with $0.3 \%$ hydrogen peroxide in PBS for $20 \mathrm{~min}$ at $37^{\circ} \mathrm{C}$ to inactivate endogenous peroxidase. Cells were then incubated in TUNEL detection solution (50 $\mu \mathrm{l} /$ well; Beyotime Institute of Biotechnology) for $60 \mathrm{~min}$ at $37^{\circ} \mathrm{C}$ and $50 \mu \mathrm{l}$ streptavidin-horseradish peroxidase solution for $30 \mathrm{~min}$. Cells were visualized by 3,3'-diaminobenzidine tetrahydrochloride (DAB) solution (Solarbio). The nucleus was stained with hematoxylin in $20 \mathrm{~min}$ at $37^{\circ} \mathrm{C}$.

Singlet oxygen detection. Alterations in singlet oxygen were detected using 2'-7'-dichlorodihydrofluorescein diacetate (DCFH-DA; Beyotime Institute of Biotechnology) according to the manufacturer's instruction. Briefly, $2 \times 10^{5}$ cells per well were seeded in 6-well plates. After incubation for $12 \mathrm{~h}$ at $37^{\circ} \mathrm{C}$, the cells were treated with hematoporphyrin $(85 \mathrm{nM}$, the $\left.\mathrm{IC}_{50}\right)$ under light irradiation $\left(625 \mathrm{~nm}, 5 \mathrm{~mW} / \mathrm{cm}^{2}\right)$ for $60 \mathrm{~min}$. The untreated cells served as the control. Subsequently, cells were cultured for $6 \mathrm{~h}$ and incubated with $10 \mu \mathrm{M}$ DCFH-DA for $30 \mathrm{~min}$ in the dark. The cells were then washed with FBS-free Dulbecco's modified Eagle's medium (Life, Thermo Fisher Scientific, Inc., Waltham, MA, USA), collected with trypsin-EDTA and resuspended in PBS. The absorbance values were detected using a microplate reader (using an 
emission wavelength of $488 \mathrm{~nm}$ and an excitation wavelength of $525 \mathrm{~nm}$. The experiments were independently performed three times.

Mitochondrial membrane potential analysis. Alterations in mitochondrial membrane potential were detected using JC-1 kit (C2005; Beyotime Institute of Biotechnology, Beijing, China) according to the manufacturer's instructions. Briefly, $2 \times 10^{5}$ cells per well were seeded in 6-well plates. Following incubation for $12 \mathrm{~h}$ at $37^{\circ} \mathrm{C}$, the cells were treated with hematoporphyrin $\left(85 \mathrm{nM}\right.$, the $\left.\mathrm{IC}_{50}\right)$ under light irradiation $\left(625 \mathrm{~nm}, 5 \mathrm{~mW} / \mathrm{cm}^{2}\right)$ for $60 \mathrm{~min}$. The untreated cells served as the control. Cells were incubated with $1 \mathrm{ml}$ work solution of JC-1 for $20 \mathrm{~min}$ at $37^{\circ} \mathrm{C}$ in the dark, washed three times with JC-1 buffer, collected with trypsin-EDTA, resuspended in PBS and detected by inverted fluorescence microscopy. JC-1 dye exhibits potential-dependent accumulation in mitochondria, which is indicated by a fluorescence emission shift from green $(\sim 529 \mathrm{~nm})$ to red $(\sim 590 \mathrm{~nm})$. Consequently, mitochondrial depolarization is indicated by a decrease in the red/green fluorescence intensity ratio. Carbonyl cyanide m-chlorophenylhydrazone (CCCP) served as positive controls (in the JC-1 kit, Beyotime Institute of Biotechnology). The ratios of the absorbance values at 525 and $590 \mathrm{~nm}$ were determined with a microplate reader. The experiments were independently performed three times.

Mature miRNA microarray analysis. The four groups, which included untreated glioma cells (in the dark; $n=3$; named Control-dark group), glioma cells treated with hematoporphyrin and without light irradiation $(\mathrm{n}=3$; named Hep-dark group), glioma cells that underwent light irradiation ( $\mathrm{n}=3$ named Control-light group), and glioma cells treated with hematoporphyrin and light irradiation $(n=3$; named Hep-light group), were analyzed using Agilent Human miRNA microarray chips $(8 \times 60 \mathrm{~K})$ V21.0 [ShanghaiBio Corp. (SBC), Shanghai, China]. The differentially expressed miRNAs that exhibited a two-fold or greater change were screened.

Reverse transcription-quantitative polymerase chain reaction $(R T-q P C R)$. Glioma cells were treated with or without PDT and their total RNA was separately extracted using an RNA extraction kit (cat. no. DP431; Tiangen Biotech Co., Ltd., Beijing, China) according to the manufacturer's instructions. The RNA concentration was quantified using an ultraviolet spectrophotometer (NanoDrop 2000; Thermo Fisher Scientific, Inc.) and was reverse transcribed into cDNA using a FastQuant first strand cDNA synthesis kit (cat. no. KR104; Tiangen Biotech Co., Ltd.) and T100 thermal Cycler for PCR (Bio-Rad Laboratories, Inc., Hercules, CA, USA). The sequences of specific primers for each gene (Guangzhou IGE Biotechnology Ltd., Guangzhou, China) were as follows: hsa-miR-205-5p, 5'-CCTTCATTC CACCGGAGTCTGAA-3'; hsa-miR-21-5p, 5'-GCCGTAGCT TATCAGACTGATGTTGAA-3'; hsa-miR-7641, 5'-TGCCGT TGATCTCGGAAGCTAAG-3'; hsa-miR-9500, 5'-CCAAGG GAAGATGGTGACCACAA-3'; hsa-miR-135b-5p, 5'-TGC TATGGCTTTTCATTCCTATGTGAA-3'; hsa-miR-210-3p, 5'-GCGTGTGACAGCGGCTGAAA-3'; hsa-miR-30b-5p, 5'-TGCCCGTGTAAACATCCTACACTCA-3'; hsa-miR-4459, 5'-GAGGCGGAGGAGGTGGAGAAA-3'; hsa-miR-663a,
5'-GCGCCGCGGGACCGCAA-3'; U6, 5'-CTCGCTTCG GCAGCACA-3'; hsa-miR-1273e, 5'-TGAACCCAGGAAGTG GAAAAA-3'; hsa-miR-642a-3p, 5'-AGACACATTTGGAGA GGGAACC-3'; hsa-miR-6893-5p, 5'-CAGGCAGGTGTA GGGTGGAG-3'; hsa-miR-7641, 5'-TGTTGATCTCGGAAG CTAAGCA-3'; hsa-miR-135b-5p, 5'-CCTATGGCTTTTCAT TCCTATGTG-3'; hsa-miR-210-3p, 5'-AAACTGTGCGTG TGACAGCG-3'; hsa-miR-30b-5p, 5'-TCCTGTAAACAT CCTACACTCAGCTA-3'; hsa-miR-31-5p, 5'-TAGGCAAGA TGCTGGCATAGAA-3'. qPCR analysis was performed in a real-time PCR thermocycler (CFX96 real-time PCR; Bio-Rad Laboratories, Inc.) using the enhanced miRNA SYBR-Green RT-PCK kit (cat. no. FP411; Biotech Co., Ltd.) and run for 40 cycles at $95^{\circ} \mathrm{C}$ for $20 \mathrm{sec}$ and $60^{\circ} \mathrm{C}$ for $34 \mathrm{sec}$. The relative miRNA levels were normalized to that of U6 using the $2^{-\Delta \Delta C q}$ method (24). The experiment was performed three times.

Gene Ontology $(G O)$ and pathway enrichment analyses. The differentially expressed miRNAs were further analyzed for predicted gene targets simultaneously using at least two of the following five databases: TargetMiner, miRDB, microRNA, TarBase and RNA22 via the SBC analysis system (http://sas.ebioservice.com). GO enrichment analyses and Kyoto Encyclopedia of Genes and Genomes (KEGG) pathway enrichment analyses were performed by the SBC analysis system, which uses clusterProfiler data from $\mathrm{R}$ and Bioconductor software (http://www.r-project.org and http://www.bioconductor.org/) with public databases that include NCBIEntrez Gene (http://www.ncbi.nlm.nih.gov/gene), GO (http://www.geneontology.org),KEGG (http://www.genom e.jp/kegg) and BioCarta (http://www.biocarta.com). The hypergeometric test was employed with a Benjamini-Hochberg-False Discovery Rate-based multiple testing correction (correction, $\mathrm{P}<0.01)(25)$.

Protein array detection assay. A Human Apoptosis Antibody Array (cat. no. AAH-APO-1; RayBiotech, Inc., Norcross, GA, USA) was used according to the manufacturer's instructions. Following development, films were scanned using ImageQuant LAS 4000 (GE Healthcare, Chicago, IL, USA) and the images were processed and quantified using the AAH-APO-1 software.

Statistical analysis. Statistical analysis was performed using GraphPad Prism Software (version 6.0; GraphPad Software, Inc., La Jolla, CA, USA) and Student's t-test. Each experiment was performed at least three times and all figures represent means \pm standard deviation. $\mathrm{P}<0.05$ was considered to indicate a statistically significant difference.

\section{Results}

PDT with PS, hematoporphyrin inhibits cell viability in a dose-dependent manner. The cell viability in U87 and U251 glioma cells following PDT was detected with PS, hematoporphyrin (Fig. 1). In the U87 and U251 cells (Fig. 1A), cell viability was significantly decreased along with the hematoporphyrin concentration. The level of cell viability was reduced to a greater extent in the U87 cells than in the U251 

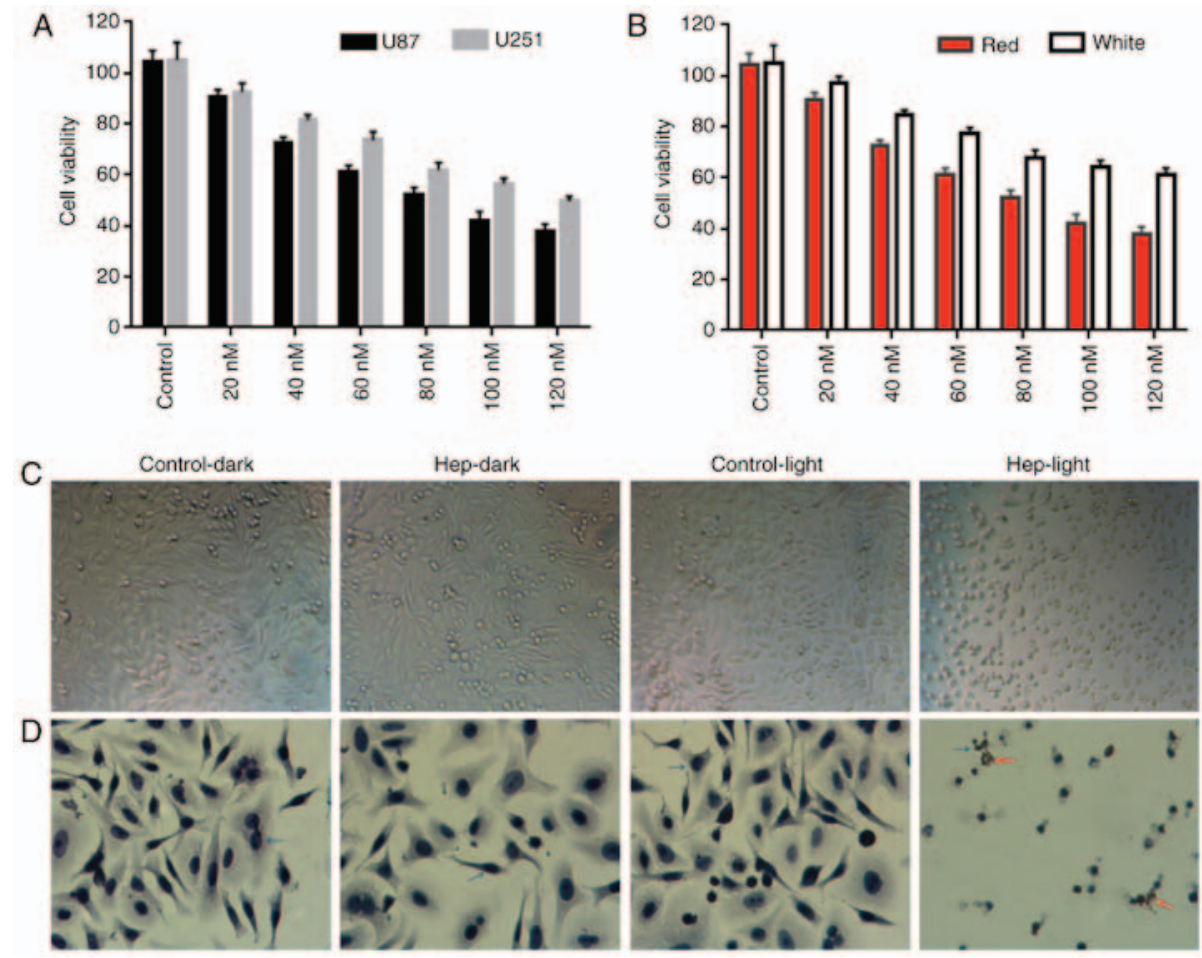

Figure 1. Effect of PDT with photosensitizer, hematoporphyrin in cell viability of glioma cells. (A) Cell viability of U87 and U251 cells following PDT treatment with photosensitizer, hematoporphyrin. (B) The viability of U87 cells irradiated under white or red light. (C) Effect of photodynamic activity of hematoporphyrin $(85 \mathrm{nM})$ in U87 cell morphology under red light. (D) Cell apoptosis was detected by TUNEL assay. Following hematoxylin staining, the nuclei were stained blue/purple (blue arrows). Following hematoporphyrin treatment with light irradiation, the nuclei were stained brown by 3,3'-diaminobenzidine tetrahydrochloride, indicating apoptotic cells (red arrow). Hep-light, cells treated with hematoporphyrin and light irradiation; Control-dark, untreated cells; Hep-dark, cells treated with hematoporphyrin; Control-light, cells treated by light irradiation. PDT, photodynamic therapy.

cells $\left(\mathrm{IC}_{50}: 85\right.$ vs. $166 \mathrm{Nm}$ for U87 vs. U251), indicating that the inhibition of PDT in U87 is better than in U251. The viability of cells that irradiated with white or red light in the U87 cells was compared (Fig. 1B) and the results demonstrated that the inhibition of hematoporphyrin under red light was better than under white light. Thus, red light-irradiated U87 cells were used in the following experiments.

Photodynamic activity of hematoporphyrin $(85 \mathrm{nM})$ induced changes in cell morphology in the U87 cells (Fig. 1C and D). The U87 cells demonstrated morphological changes following PDT-hematoporphyrin treatment, including shrinking, fragmentation, which was not observed in cells without hematoporphyrin treatment (in the light or dark; Fig. 1C and D).

PDT with PS, hematoporphyrin induces cell apoptosis. Cell apoptosis in U87 cells following PDT with PS, hematoporphyrin $(85 \mathrm{nM})$ were detected by Hoechst 33342 and flow cytometry (Fig. 2). As presented in Fig. 2A, photodynamic activity of hematoporphyrin induced apoptotic nuclei in U87 cells with low cell density. Similarly, the flow cytometric analysis revealed that photodynamic activity of hematoporphyrin increased cell apoptosis in early (6.1 vs. $5.91 \%$ for PDT vs. control) and later (26.35 vs. $0.44 \%$ for PDT vs. control; $\mathrm{P}<0.05$ ) stages (Fig. 2B). Similar to the Hoechst 33342 and flow cytometry data, the TUNEL assay indicated that the photodynamic activity of hematoporphyrin increased cell apoptosis (Fig. 1D). Thus, photodynamic activity of hematoporphyrin inhibited cell growth via induction of cell apoptosis.
PDT with PS, hematoporphyrin induces cell apoptosis via induction of ROS. As previous studies demonstrated that, under light irradiation, hematoporphyrin exerts its action via production of singlet oxygen and superoxide anions $(26,27)$, the current study investigated the alterations in singlet oxygen and mitochondrial membrane potential in cells following PDT with PS, hematoporphyrin treatment (Fig. 3). In healthy cells, the mitochondrial membrane potential was not markedly changed, while obvious green fluorescence was observed in the U87 cells following PDT treatment (Fig. 3A). A similar result was obtained using a microplate reader, the mitochondrial membrane potential was significantly decreased following PDT treatment, which was lower than the CCCP group (Fig. 3B). The content of reactive oxygen species (ROS) was significantly increased by PDT, which was greater than that in the Rosup group (Fig. 3C). Thus, PDT with hematoporphyrin may result in the induction of ROS and the decrease in mitochondrial membrane potential, which are important in cell apoptosis and mitochondrial disorders (28).

Screening and validation of miRNA involved in PDT with PS, hematoporphyrin-induced cell apoptosis. The potential target miRNA was analyzed by miRNA microarray, and the differentially expressed miRNAs between Hep-light (cells with hematoporphyrin and light irradiation) and Control-dark (cells without treatment), Hep-dark (cells with hematoporphyrin) or Hep-light (cells with light irradiation) groups were screened. There were 185 miRNAs differentially expressed in the PDT treatment groups compared with the other groups. 

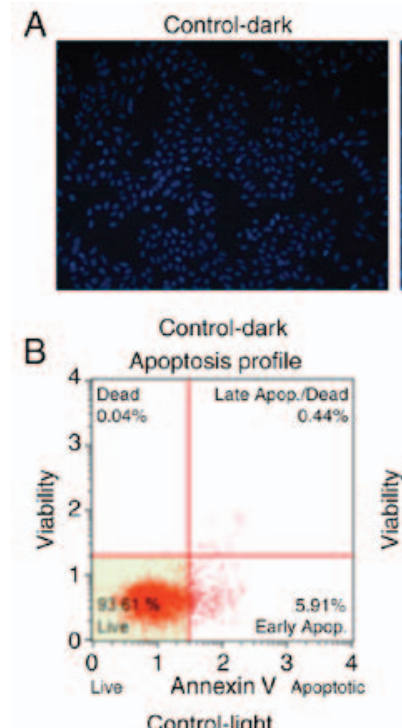

Control-light

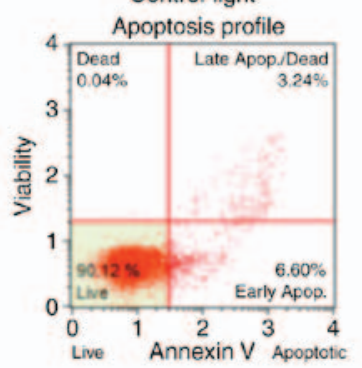

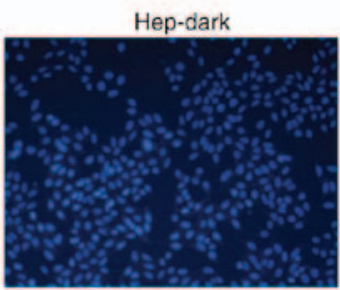

Hep-dark

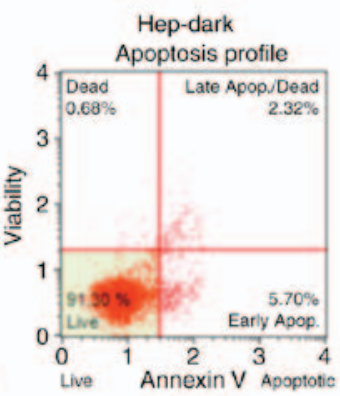

Hep-light

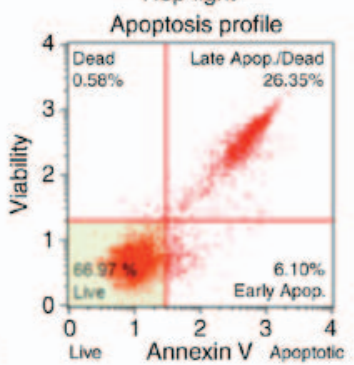

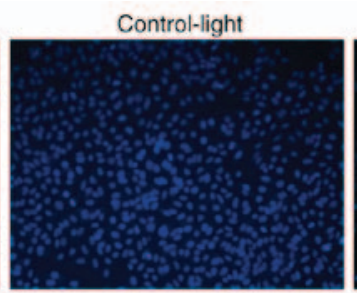
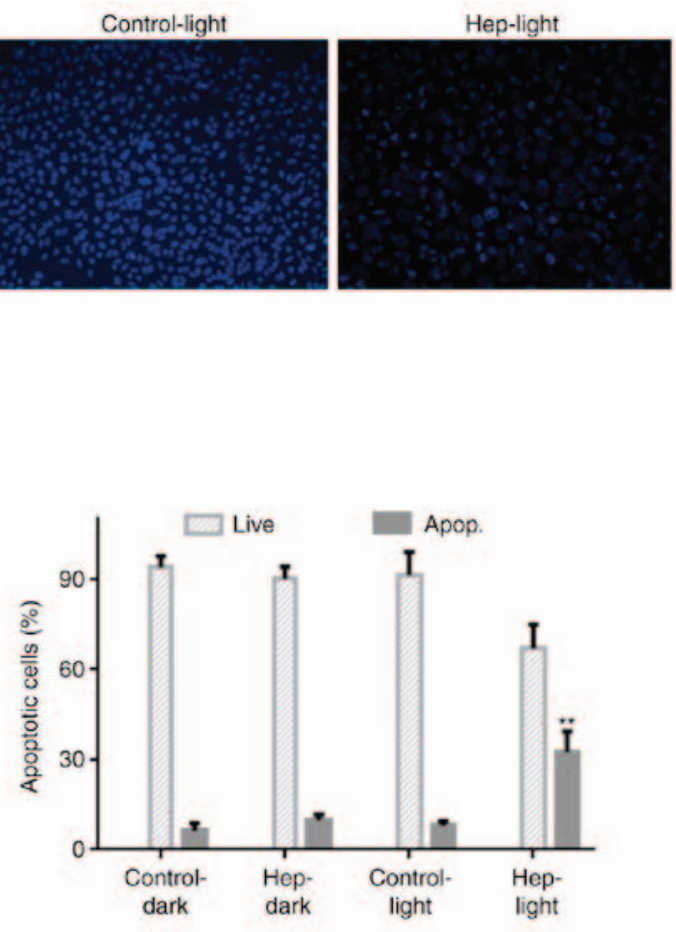

Figure 2. Effect of photodynamic therapy with photosensitizer, hematoporphyrin in cell apoptosis of U87 cells. (A) Effect of photodynamic activity of hematoporphyrin on apoptotic nuclei in U87 cells; magnification, x200. (B) Effect of photodynamic activity of hematoporphyrin on U87 cell apoptosis detected by flow cytometry and analysis by FlowJo software. Hep-light, cells treated with hematoporphyrin and light irradiation; Control-dark, untreated cells; Hep-dark, cells treated with hematoporphyrin; Control-light, cells treated by light irradiation. ${ }^{* *} \mathrm{P}<0.01$, significantly different compared with the control by $\mathrm{t}-\mathrm{test}$, $\mathrm{n}=3$.
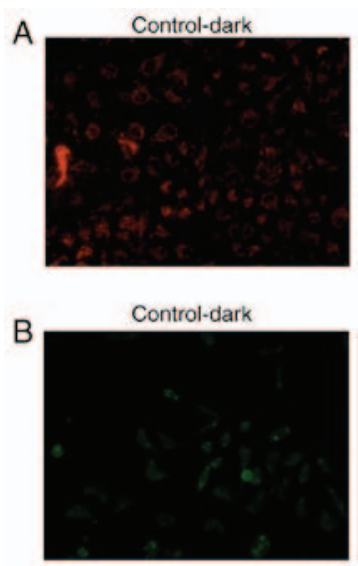

C
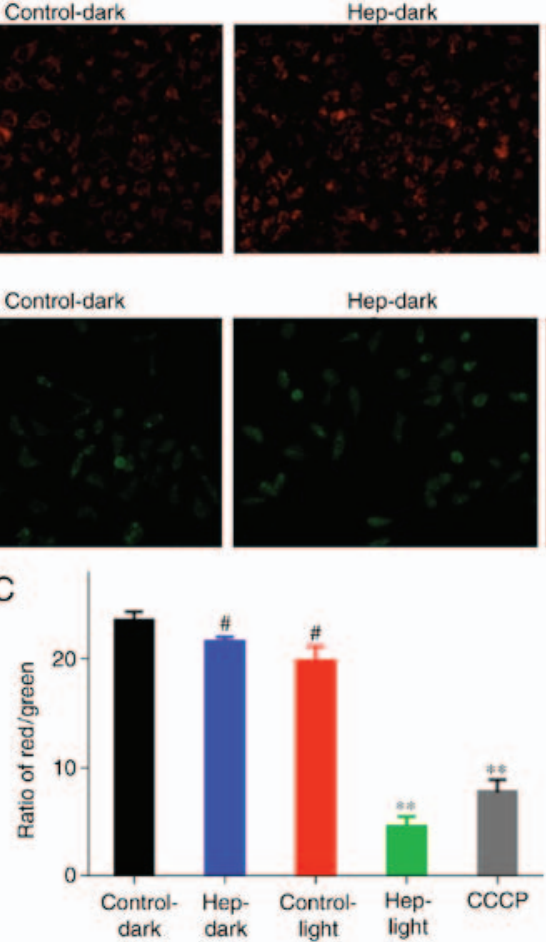

Hep-dark

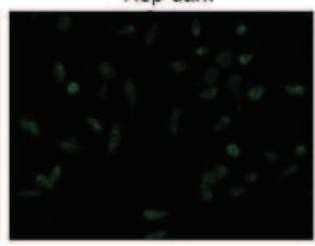

Control-light

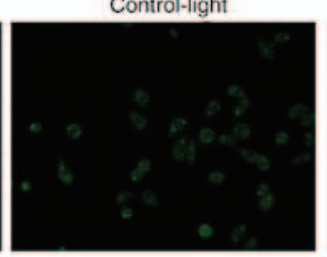

D

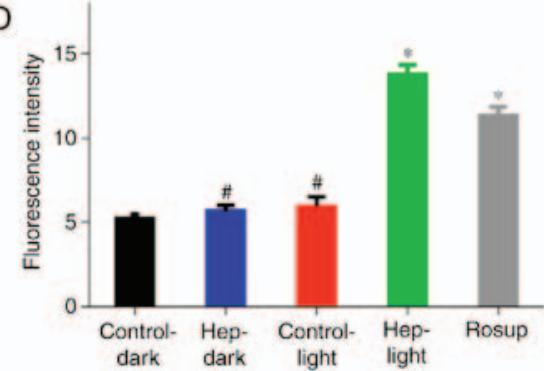

Figure 3. Mitochondrial membrane potential and reactive oxygen species content in U87 cells treated with PDT and hematoporphyrin. (A and B) JC-1 dye with emissions of $590 \mathrm{~nm}$ (red) and $525 \mathrm{~nm}$ (green). (C) Mitochondrial depolarization is indicated by a decrease in the red/green fluorescence intensity ratio. (D) Singlet oxygen was detected using 2'-7'-dichlorodihydrofluorescein diacetate. CCCP and Rosup served as positive controls. Hep-light, cells treated with hematoporphyrin and light irradiation; Control-dark, untreated cells; Hep-dark, cells treated with hematoporphyrin; Control-light, cells treated by light irradiation. ${ }^{*} \mathrm{P}<0.05,{ }^{* *} \mathrm{P}<0.01$ and ${ }^{\#} \mathrm{P}>0.01$ vs. the control (untreated cells) (Student's t-test; $\mathrm{n}=3$ ). CCCP, carbonyl cyanide m-chlorophenylhydrazone. 

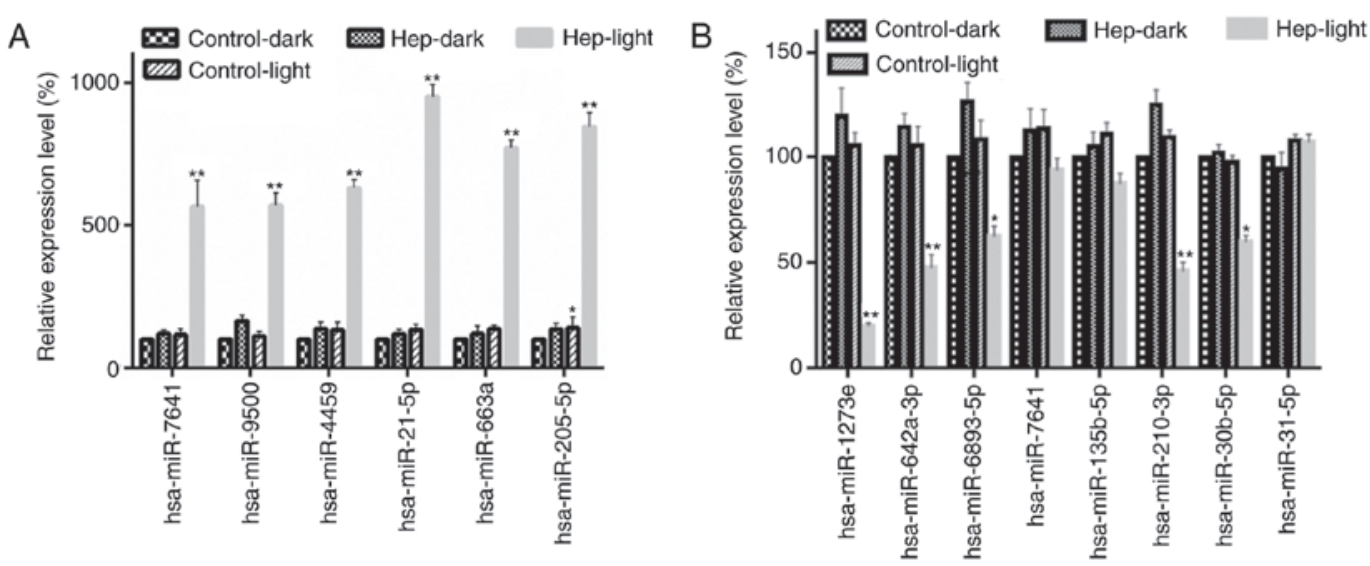

Figure 4. Validation of differentially expressed miRNAs obtained from miRNA microarray analysis in U87 cells. The differentially expressed miRNAs were confirmed by reverse transcription-quantitative polymerase chain reaction. (A) Upregulated and (B) downregulated miRNAs. Hep-light, cells treated with hematoporphyrin and light irradiation; Control-dark, untreated cells; Hep-dark, cells treated with hematoporphyrin; Control-light, cells treated by light irradiation. ${ }^{* *} \mathrm{P}<0.01$ vs. the control (untreated cells) (Student's t-test; $\mathrm{n}=3$ ). miRNA, microRNA.

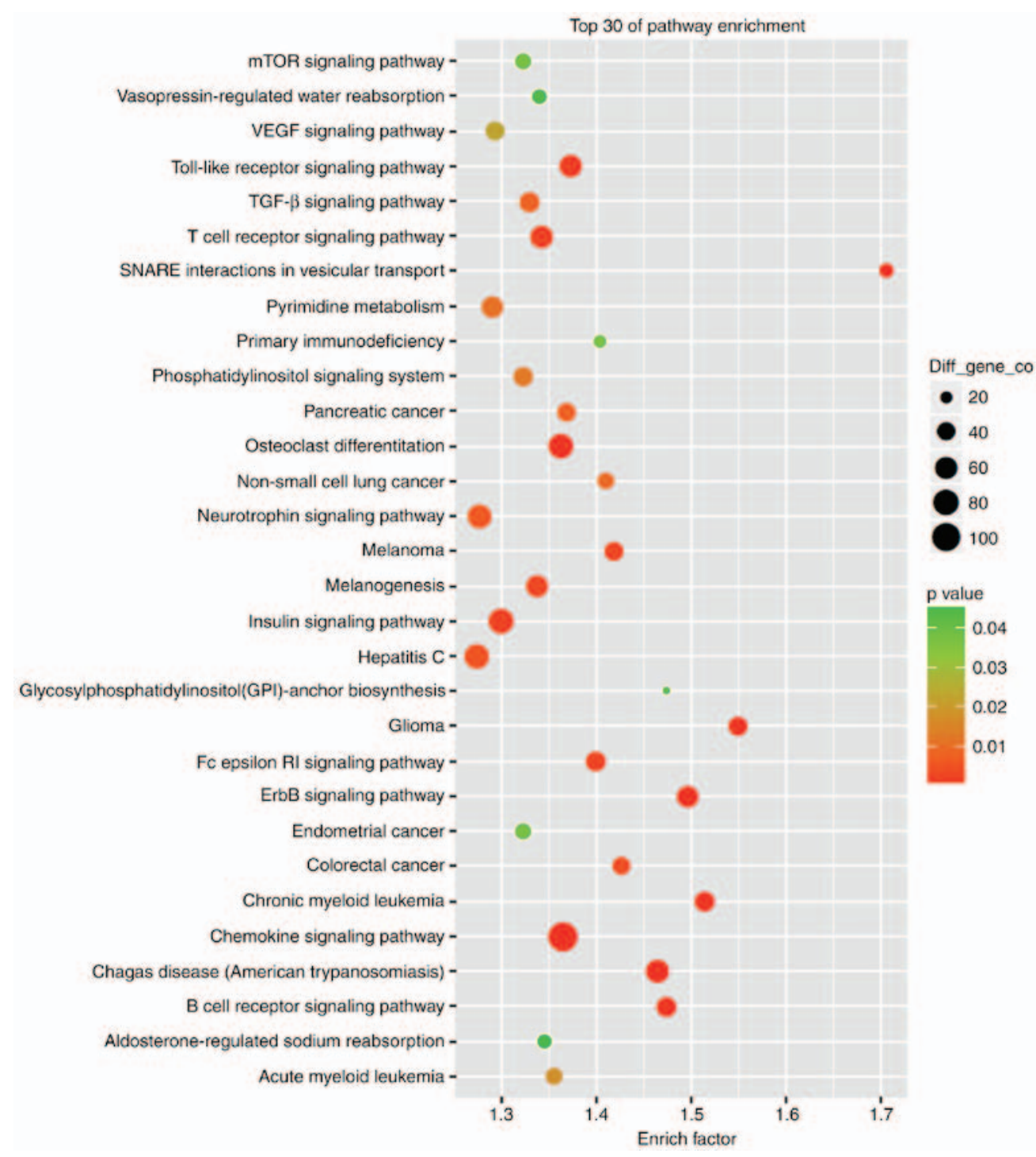

Figure 5. Gene Ontology and Kyoto Encyclopedia of Genes and Genomes enrichment analysis of differentially expressed microRNAs in U87 cells.

The top six upregulated miRNAs included hsa-miR-7641, hsa-miR-9500, hsa-miR-4459, hsa-miR-21-5p, hsa-miR-663a and hsa-miR-205-5p. The top eight downregulated miRNAs included hsa-miR-1273e, hsa-miR-642a-3p, hsa-miR-6893-5p, hsa-miR-7641, hsa-miR-135b-5p, hsa-miR-210-3p, hsa-miR-30b-5p and hsa-miR-31-5p. The expression levels 

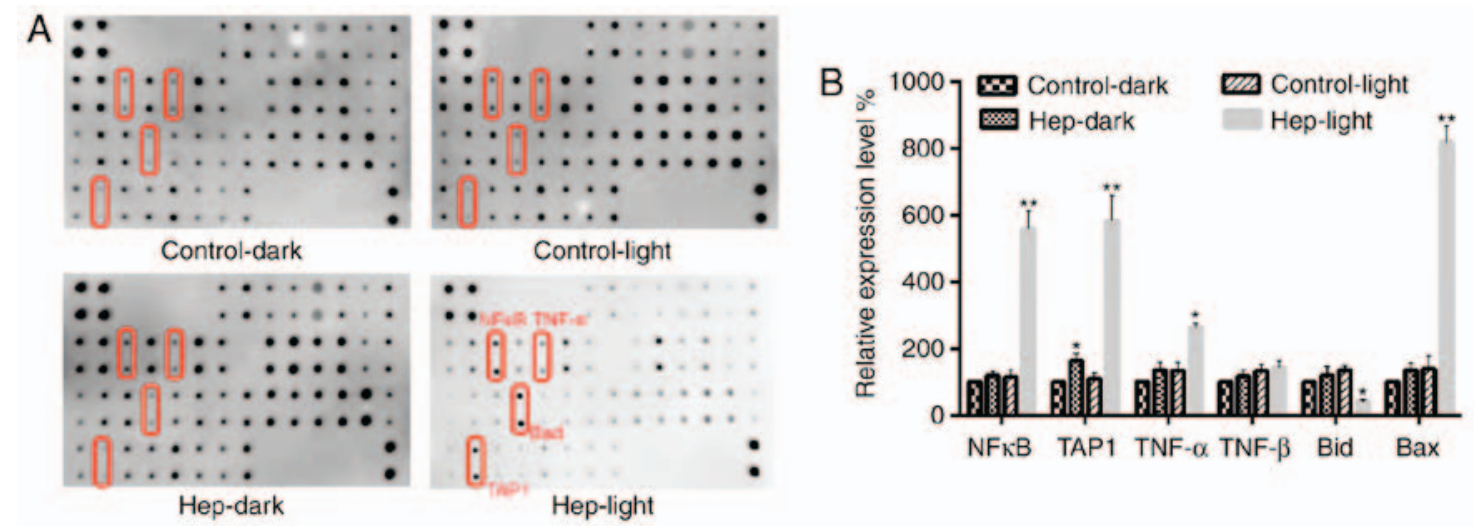

Figure 6. Detection of cell apoptosis-associated proteins using apoptosis antibody array in U87 cells. (A) Films were scanned using ImageQuant LAS 4000. (B) Quantification data of differentially expressed proteins. Hep-light, cells treated with hematoporphyrin and light irradiation; Control-dark, untreated cells; Hep-dark, cells treated with hematoporphyrin; Control-light, cells treated by light irradiation. ${ }^{*} \mathrm{P}<0.05$ and ${ }^{* *} \mathrm{P}<0.01$ vs. the control (untreated cells) (Student's

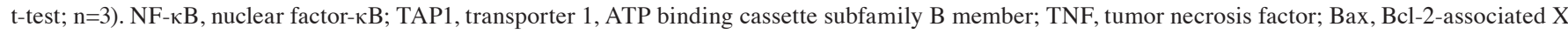
protein.

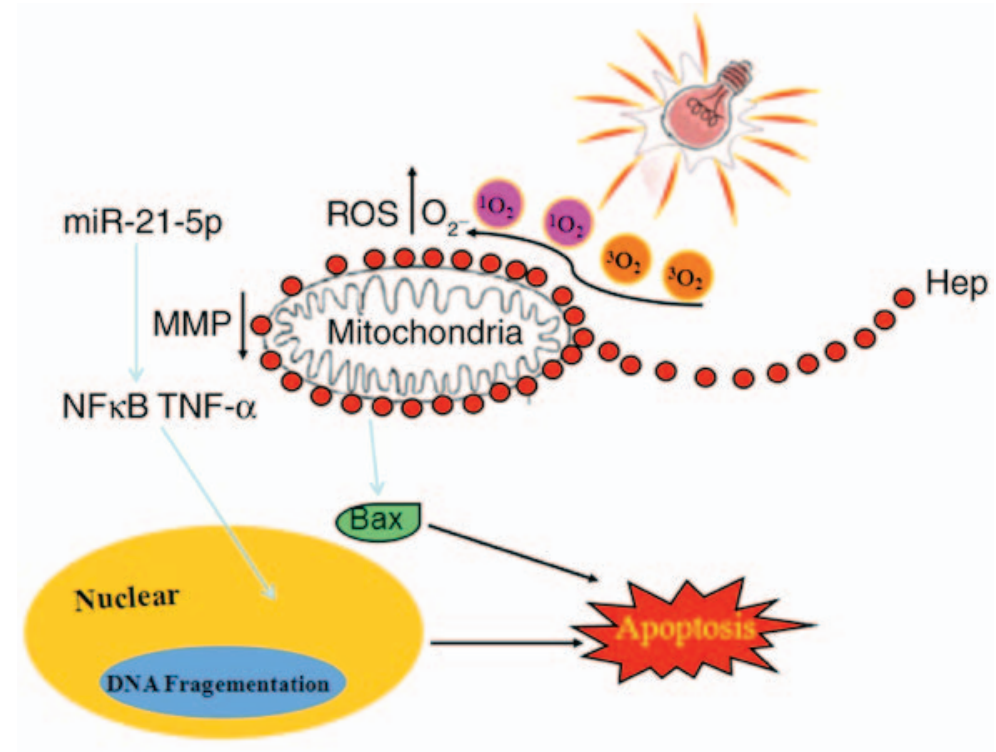

Figure 7. Proposed mechanistic of hematoporphyrin (Hep)-induced apoptosis in U87 cells under irradiation. Differentially expressed miRNAs, such as hsa-miR-21-5p, are involved in PDT-inhibited glioma cell growth via induction of cell apoptosis and necrosis. Following PDT with hematoporphyrin treatment in U87 cells, the expression levels of NF- $\mathrm{kB}$, TAP1, Bax, and TNF- $\alpha$ were significantly upregulated, and the expression level of Bid was significantly downregulated. The TAP1 and NF-kB-mediated apoptosis pathway was the most important pathway involved in PDT with hematoporphyrin-induced apoptosis in glioma U87 cells. It indicates that the miRNAs that specifically targeted TAP1 and NF- $\mathrm{kB}$ are important therapeutic targets. miRNA, microRNA; PDT, photodynamic therapy; NF-kB, nuclear factor- $\mathrm{kB}$; TAP1, transporter 1, ATP binding cassette subfamily B member; TNF, tumor necrosis factor; Bax, Bcl-2-associated X protein; ROS, reactive oxygen species; MMP, matrix metalloproteinase; Hep, haematoporphyrin.

of the six upregulated miRNAs, including hsa-miR-7641, hsa-miR-9500, hsa-miR-4459, hsa-miR-21-5p, hsa-miR-663a and hsa-miR-205-5p were confirmed by RT-qPCR (Fig. 4A). In addition, the expression levels of the top eight downregulated miRNAs were detected by RT-qPCR (Fig. 4B). The expression levels of hsa-miR-1273e, hsa-miR-642a-3p, hsa-miR-6893-5p, hsa-miR-210-3p and hsa-miR-30b-5p were significantly downregulated in the PDT treatment groups, compared with in the other groups.

GO and KEGG enrichment analysis demonstrated that the most significant cellular functions were cell apoptosis, cell necrosis and ROS-mediated functions, and the most significant signaling pathways were the chemokine signaling pathway, osteoclast differentiation and the insulin signaling pathway, indicating that PDT inhibited glioma cell growth via induction of cell apoptosis and necrosis (Fig. 5). In the process, the upregulated miRNAs, including hsa-miR-1273e, hsa-miR-642a-3p, hsa-miR-6893-5p, hsa-miR-7641, hsa-miR-9500, hsa-miR-135b-5p, hsa-miR-205-5p, hsa-miR-21-5p, hsa-miR-210-3p, hsa-miR-30b-5p, hsa-miR-31-5p, hsa-miR-4459 and hsa-miR-663a are significant and represent important targets for therapy of PDT-induced cell apoptosis in glioma.

Detection of cell apoptosis-associated proteins. The proteins associated with cell apoptosis were detected by apoptosis antibody array (Fig. 6). Quantitative analysis revealed that TAP1- and nuclear factor (NF)- $\mathrm{KB}$-mediated apoptosis 
pathways were the most important signaling pathways involved in PDT with hematoporphyrin-induced apoptosis observed in glioma U87 cells (Fig. 6A). Following PDT with hematoporphyrin treatment in U87 cells, the expression levels of NF- $\kappa$ B, TAP1, Bcl-2-associated $\mathrm{X}$ protein and tumor necrosis factor- $\alpha$ were significantly upregulated, and the expression level of Bid was significantly downregulated $(\mathrm{P}<0.05$; Fig. $6 \mathrm{~B})$.

\section{Discussion}

The current study examined the possibility of using PDT for the treatment of gliomas, and evaluated the molecular mechanism of PDT using hematoporphyrin as a PS for the inhibition of glioma cell growth by screening target miRNAs and apoptosis-associated proteins. The aim of the study was to provide a theoretical reference for the design and preparation of novel PSs for PDT.

Using the first generation of PS for PDT, hematoporphyrin on U251 and U87 glioma cells, the inhibition of PDT in U87 cells was identified to be better than that in U251 cells. Under the same conditions, the $\mathrm{IC}_{50}$ of hematoporphyrin in the U87 cells was $80 \mathrm{nM}$, while that in the U251 cells was $120 \mathrm{nM}$. Physical and chemical factors act on cells together (29), and PS and light source are two key factors of PDT. Therefore, the effect of single wavelength red light and non-single wavelength white light on photodynamic activity of hematoporphyrin were detected by irradiation of U87 cells. The results demonstrated that the inhibition of hematoporphyrin under red light was better than that under white light. Thus, red light-irradiated U87 cells were used to investigate the molecular mechanism.

The inhibition of cell growth by drugs is generally caused by induction of apoptosis or cell cycle arrest (23). The photodynamic activity of hematoporphyrin was found to inhibit cell growth via induction of cell apoptosis at the later stage. This is consistent with the results reported in previous studies (28). For example, the glucose-modified porphyrin derivatives, synthesized by Králová et al (30) under light irradiation significantly inhibited cell growth of MDA-MB-231 cells via induction of cell apoptosis, which was also verified in a mouse model. Thus, the PDT activity of hematoporphyrin on glioma U87 cells appears to be due to the induction of apoptosis.

It was demonstrated that hematoporphyrin under light irradiation exerts its action via production of singlet oxygen and superoxide anions $(26,27)$. The current study found that PDT treatment with hematoporphyrin induced ROS and decreased mitochondrial membrane potential, which contributed to mitochondrial disorder.

Previous studies demonstrate that apoptosis is closely regulated by miRNAs $(31,32)$. The potential target miRNA was analyzed by miRNA microarray and differentially expressed miRNAs, including six upregulated and eight downregulated miRNAs between Hep-light (cells with hematoporphyrin and light irradiation) and Control-dark (cells without treatment), Hep-dark (cells with hematoporphyrin), or Hep-light (cells with light irradiation) groups, were screened. There were 185 miRNAs differentially expressed in the PDT treatment groups when compared with other groups. The expression of six upregulated miRNAs, including hsa-miR-7641, hsa-miR-9500, hsa-miR-4459, hsa-miR-21-5p, hsa-miR-663a, and hsa-miR-205-5p were confirmed by RT-qPCR. It was reported that miR-21 regulated proliferation and apoptosis of human glioma cells by downregulating the phosphatase and tensin homolog protein (33). In a previous study, overexpression of miR-663 inhibited proliferation, migration and invasion in A172 and U87 glioblastoma cells (34). Furthermore, the miR-205 serum level was identified as an individual diagnostic marker for glioma (35). However, the roles of hsa-miR-7641, hsa-miR-9500, hsa-miR-4459, hsa-miR-21-5p, hsa-miR-663a and hsa-miR-205-5p in PDT-induced cell apoptosis have not yet bee confirmed using a cell model.

GO and KEGG enrichment analysis identified that the most significant cellular functions were cell apoptosis, cell necrosis and ROS-mediated functions, and the most significant signaling pathways were the chemokine signaling pathway, osteoclast differentiation and the insulin signaling pathway, indicating that PDT inhibited glioma cell growth via induction of cell apoptosis and necrosis. The proteins associated with cell apoptosis were detected by apoptosis antibody array, and quantitative analysis revealed that TAP1 and NF- $\kappa \mathrm{B}$-mediated apoptosis signaling pathway was the most important pathway involved in PDT, with hematoporphyrin-induced apoptosis observed in glioma U87 cells. A recent study reported that miR-21 significantly contributes to the inflammatory response via the sirtuin $1-\mathrm{NF}-\kappa \mathrm{B}$ signaling pathway (36). It implied that the miRNAs that specifically targeted TAP1 and NF- $\kappa \mathrm{B}$ are important therapeutic targets (Fig. 7). However, whether changes of AP1- and NF-kB are associated with those miRNAs remains unclear.

TAPl and NF-KB proteins may be important in the apoptotic process. Furthermore, 5-ALA may significantly inhibit the growth of glioma U251 cells under light irradiation, and the expression levels of TAP1-associated proteasomes has been demonstrated to be significantly increased (20). In addition, PDT was demonstrated to induce phosphorylation of proteins in the Bcl-2 family, enhancing the intracellular oxidative stress response and thereby accelerating apoptosis of U87 cells (37). According to the results of a previous study and previous clinical experience $(38,39)$, PDT was hypothesized to be a potential treatment strategy for glioma via apoptosis induction and immune enhancement (19-22), which was confirmed in the current study. PDT represents a potential treatment strategy for glioma, and the treatment effect appears to be more obvious under a red light source. PDT inhibited cell growth via induction of cell apoptosis and immune enhancement. PDT treatment with hematoporphyrin inhibits cell growth of gliomas, which is regulated by miRNAs; however, the target genes for miRNAs and the molecular network remain unclear. Therefore, further studies are required in future. In the current study, the experiments, such as cell apoptosis, were only performed at one time point ( $23 \mathrm{~h}$ post-PDT treatment); thus, further research is required to verify the mechanism of action at additional time points. Furthermore, in this study, the human glioma cell lines, U87 and U251, were used; in future studies, we also aim to use normal control primary glioma cells from rats or mice to further confirm our findings.

\section{Acknowledgements}

The present study was supported by the Natural Science Foundation of China (grant no. 81272774). 


\section{Competing interests}

The authors declare that they have no competing interests.

\section{References}

1. Duan R, Han L, Wang Q, Wei J, Chen L, Zhang J, Kang C and Wang L: HOXA13 is a potential GBM diagnostic marker and promotes glioma invasion by activating the Wnt and TGF- $\beta$ pathways. Oncotarget 6: 27778-27793, 2015.

2. Watkins S, Robel S, Kimbrough IF, Robert SM, Ellisdavies G and Sontheimer H: Disruption of astrocyte-vascular coupling and the blood-brain barrier by invading glioma cells. Nature Communications 5: 4196, 2014.

3. Li W and Graeber MB: The molecular profile of microglia under the influence of glioma. Neuro Oncol 14: 958-978, 2012.

4. Saito T, Muragaki Y, Maruyama T, Tamura M, Nitta M and Okada Y: Intraoperative functional mapping and monitoring during glioma surgery. Neurol Med Chir 55 (Suppl 1): S1-S13, 2015.

5. ShiM,FortinD,SancheLandPaquetteB:Convection-enhancement delivery of platinum-based drugs and Lipoplatin ${ }^{\mathrm{TM}}$ to optimize the concomitant effect with radiotherapy in F98 glioma rat model. Invest New Drugs 33: 555-563, 2015.

6. Tobias A, Ahmed A, Moon KS and Lesniak MS: The art of gene therapy for glioma: A review of the challenging road to the bedside. J Neurol Neurosurg Psychiatry 84: 213-222, 2013.

7. Charest G, Sanche L, Fortin D, Mathieu D and Paquette B Optimization of the route of platinum drugs administration to optimize the concomitant treatment with radiotherapy for glioblastoma implanted in the Fischer rat brain. J Neurooncol 115 365-373, 2013

8. Rudà R, Bello L, Duffau H and Soffietti R: Seizures in low-grade gliomas: Natural history, pathogenesis, and outcome after treatments. Neuro Oncol 14 (Suppl 4): iv55-iv64, 2012.

9. Ethirajan M, Chen Y, Joshi P and Pandey RK: The role of porphyrin chemistry in tumor imaging and photodynamic therapy. Chem Soc Rev 40: 340-362, 2011.

10. Dougherty TJ, Gomer CJ, Henderson BW, Jori G, Kessel D, Korbelik M, Moan J and Peng Q: Photodynamic therapy. J Natl Cancer Inst 90: 889-905, 1998.

11. Krosl G, Korbelik M and Dougherty GJ: Induction of immune cell infiltration into murine SCCVII tumour by photofrin-based photodynamic therapy. Br J Cancer 71: 549-555, 1995.

12. Kessel D, Luo Y, Deng Y and Chang CK: The role of subcellular localization in initiation of apoptosis by photodynamic therapy. Photochem Photobiol 65: 422-426, 1997.

13. Dougherty TJ and Levy JG: Biomedical photonics handbook. CRC Press, New York, 2003.

14. Tian YY, Wang LL and Wang W: Progress in photodynamic therapy on tumors. Laser Physics 18: 1119-1123, 2008.

15. Sutedja T, Baas P, Stewart F and van Zandwijk N: A pilot study of photodynamic therapy in patients with inoperable non-small cell lung cancer. Eur J Cancer 28A: 1370-1373, 1992.

16. Pandey RK, Goswami LN, Chen Y, Gryshuk A, Missert JR, Oseroff A and Dougherty TJ: Nature: A rich source for developing multifunctional agents. Tumor-imaging and photodynamic therapy. Lasers Surg Med 38: 445-467, 2006.

17. Whelpton R, Michael-Titus AT, Basra SS and Grahn M: Distribution of temoporfin, a new photosensitizer for the photodynamic therapy of cancer, in a murine tumor model. Photochem Photobiol 61: 397-401, 1995.

18. Ji J, Fan Z, Zhou F, Wang X, Shi L, Zhang H, Wang P, Yang D, Zhang L, Chen WR and Wang X: Improvement of DC vaccine with ALA-PDT induced immunogenic apoptotic cells for skin squamous cell carcinoma. Oncotarget 6: 17135-17146, 2015.

19. Wen CC, Li JL, Zhang SY, Xu XK, Ouyang LP, Yu J and Li FC: Photodynamic therapy mediated with 5-aminolevulinic acid enhances the expression of proteasomes in glioma cell line U251 cells. Chin J Clin Neurosurg 17: 154-157, 2012.

20. Le-ping O, Shan-yi Z, Jun-liang L, Xin-ke X, Yin-lun W, Mei-guang Z, Sheng-wen W and Fang-cheng L: Overexpression of TAP1 up-regulates HLA-I in human glioma U251 cells. Chin J Pathophysiol 29: 425-429, 2013.
21. Yuan SX, Li FC, Zhou HJ, Sun X and Yin HJ: Antitumor efficacy of photodynamic therapy-generated glioma vaccine from dendritic cells. Tumor 27: 962-967, 2007.

22. Zhang SY, Li JL, Xu XK, Zheng MG, Wen CC and Li FC: Effect of hematoporphyrin monomethyl ether-based photodynamic treatment on gene expression of transporter associated with antigen processing 1 in human U87-MG glioma. Chin J Exp Surg 27: 914-916, 2010 (In Chinese).

23. Zhang Z, Wen JY, Lv BB, Li X, Ying X, Wang YJ, Zhang HT, Wang H, Liu HY and Chnag CK: Photocytotoxicity and G-quadruplex DNA interaction of water-soluble gallium(III) tris( $N$-methyl-4-pyridyl)corrole complex. Appl Organometal Chem 30: 132-139, 2016.

24. Liu JX, Yan ZP, Zhang YY, Wu J, Liu XH and Zeng Y: Hemodynamic shear stress regulates the transcriptional expression of heparan sulfate proteoglycans in human umbilical vein endothelial cell. Cell Mol Biol 62: 28-34, 2016.

25. Zeng Y, Liu JX, Yan ZP, Yao XH and Liu XH: Potential microRNA biomarkers for acute ischemic stroke. Int J Mol Med 36: 1639-1647, 2015.

26. Moserova I and Kralova J: Role of ER stress response in photodynamic therapy: ROS generated in different subcellular compartments trigger diverse cell death pathways. PLoS One 7: e32972, 2012

27. Broekgaarden M, Weijer R, van Gulik TM, Hamblin MR and Heger M: Tumor cell survival pathways activated by photodynamic therapy: A molecular basis for pharmacological inhibition strategies. Cancer Metastasis Rev 34: 643-690, 2015.

28. Liang ZH, Liu HY, Zhou R, Zhang Z, Ali A, Han BJ, Liu YJ and Xiao XY: DNA-binding, photocleavage, and photodynamic anti-cancer activities of pyridyl corroles. J Membr Biol 249: 419-428, 2016.

29. Zeng Y: Endothelial glycocalyx as a critical signalling platform integrating the extracellular haemodynamic forces and chemical signalling. J Cell Mol Med 21: 1457-1462, 2017.

30. Králová J, Bríza T, Moserová I, Dolenský B, Vasek P, Poucková P, Kejík Z, Kaplánek R, Martásek P, Dvorák M and Král V: Glycol porphyrin derivatives as potent photodynamic inducers of apoptosis in tumor cells. J Med Chem 51: 5964-5973, 2008.

31. Geng Y, Lin D, Shao L, Yan F and Ju H: Cellular delivery of quantum dot-bound hybridization probe for detection of intracellular pre-microRNA using chitosan/poly $(\gamma$-glutamic acid $)$ complex as a carrier. PLoS One 8: e65540, 2013.

32. Guo P, Coban O, Snead NM, Trebley J, Hoeprich S, Guo $\mathrm{S}$ and Shu Y: Engineering RNA for targeted siRNA delivery and medical application. Adv Drug Deliv Rev 62: 650-666, 2010

33. Li SJ, Zhou J, Zhang L, Xiang W, Hu Q, He YY and Chen LG: The effect of miR-21 on SWOZ2 glioma cells and its biological mechanism. J BUON 22: 468-473, 2017.

34. Li Q, Cheng Q, Chen Z, Peng R, Chen R, Ma Z, Wan X, Liu J, Meng M, Peng Z and Jiang B: MicroRNA-663 inhibits the proliferation, migration and invasion of glioblastoma cells via targeting TGF- 31 . Oncol Rep 35: 1125-1134, 2016.

35. Yue X, Lan F, Hu M, Pan Q, Wang Q and Wang J: Downregulation of serum microRNA-205 as a potential diagnostic and prognostic biomarker for human glioma. J Neurosurg 124: 122-128, 2016.

36. Lin Q, Geng Y, Zhao M, Lin S, Zhu Q and Tian Z: MiR-21 Regulates TNF- $\alpha$-induced CD40 expression via the SIRT1-NF- $\kappa$ B pathway in renal inner medullary collecting duct cells. Cell Physiol Biochem 41: 124-136, 2017.

37. Misuth M, Horvath D, Miskovsky P and Huntosova V: Synergism between PKC $\delta$ regulators hypericin and rottlerin enhances apoptosis in U87 MG glioma cells after light stimulation. Photodiagnosis Photodyn Ther 18: 267-274, 2017.

38. Pan L, Lin H, Tian S, Bai D, Kong Y and Yu L: The sensitivity of glioma cells to pyropheophorbide-amethyl ester-mediated photodynamic therapy is enhanced by inhibiting ABCG2. Lasers Surg Med 49: 719-726, 2017.

39. Christie C, Pomeroy A, Nair R, Berg K and Hirschberg H: Photodynamic therapy enhances the efficacy of gene-directed enzyme prodrug therapy. Photodiagnosis Photodyn Ther 18: 140-148, 2017. 